\title{
A systematic review and meta-analysis of randomized controlled trials exploring the role of inter-individual variability on the effect of flavanols on insulin and HOMA-IR
}

\author{
N. Alshammari ${ }^{1}$, S.A. Palma-Duran ${ }^{1}$, G. Jiang ${ }^{1}$, A. González-Sarrías ${ }^{2}$, P. Pinto $^{3}$, \\ M. Garcia-Aloy ${ }^{4}$, E. Gibney ${ }^{5}$, MT. García-Conesa ${ }^{2}$, P. Kroon ${ }^{6}$, A. Konić Ristić ${ }^{7}$, A. Katsaltou ${ }^{8}$, \\ C. Morand ${ }^{9}$, A. Rodriguez-Mateos ${ }^{10}$ and E. Combet ${ }^{1}$ \\ ${ }^{1}$ University of Glasgow, UK, ${ }^{2}$ Centro de Edafologia y Biologia Aplicada del Segura-Consejo Superior de Investigaciones \\ Cientificas, Spain, ${ }^{3}$ Polytechnic Institute of Santarem, Portugal, ${ }^{4}$ University of Barcelona, Spain, ${ }^{5}$ University College, \\ Dublin, Ireland, ${ }^{6}$ Quadram Institute, Norwich, UK, ${ }^{7}$ University of Belgrade, Serbia, ${ }^{8}$ University of Thessaly, Greece, \\ ${ }^{9}$ Institut National de la Recherche Agronomique, France and ${ }^{10}$ King's College London, UK.
}

Meta-analyses of randomised controlled trials (RCTs) report that polyphenol-rich diets can modulate a range of cardiometabolic biomarkers, with increasing evidence that inter-individual factors (e.g. age, BMI, or ethnicity) contribute toward the variability in the response to the bioactive ${ }^{(1,2)}$. This systematic review and meta-analysis assessed the effect of flavanols from cocoa, apple and tea on fasting insulin and HOMA-IR and explored the role of inter-individual variability.

PubMed and Web of Science databases were searched from inception to October 2017 (PROSPERO reg. CRD42016033878). The effect of flavanols supplementation on insulin and HOMA-IR was estimated using a random effects meta-analysis model and reported as standardised mean difference (SMD) and 95\%CI. Subgroup analyses (Q tests; multivariate meta-regression) focused on baseline $\mathrm{BMI}$, gender, age, and geographical location to explore the role of inter-individual variability.

Out of 1409 studies identified, 31 RCTs were included for insulin $(n=1792)$ and 21 RCTs for HOMA-IR $(n=1152)$. Low heterogeneity was found between studies (insulin $\mathrm{I}^{2}=0 \%, \mathrm{p}=0.98$; HOMA-IR $\mathrm{I}^{2}=5.9 \%, \mathrm{p}=0.38$ ) with evidence of low publication bias. Flavanol-rich interventions (2-26 weeks; 88 to $1344 \mathrm{mg}$ flavanols/day) decreased both insulin (SMD $-0.25,95 \%$ CI $-0.33 ;-0.16$ ) and HOMA-IR (SMD -0.26; 95\% CI -0.36, -0.16). Results were consistent across subgroups (Q tests) with lack of effect in subgroups with $\mathrm{BMI}<25$ or male subjects only; multivariate meta-regression showed that baseline BMI (overweight versus lean, coef. -1.07 ; $95 \% \mathrm{CI}-2.03,-0.08 ; \mathrm{p}=0.03$ ) and study location (Asia versus other sites, coef. $0.94 ; 95 \% \mathrm{CI} 0.03,1.84 ; \mathrm{p}=0.04$ ) impacted on the effect on HOMA-IR significantly. There was no impact of age, gender, baseline BMI or geographical location on the effect on insulin.

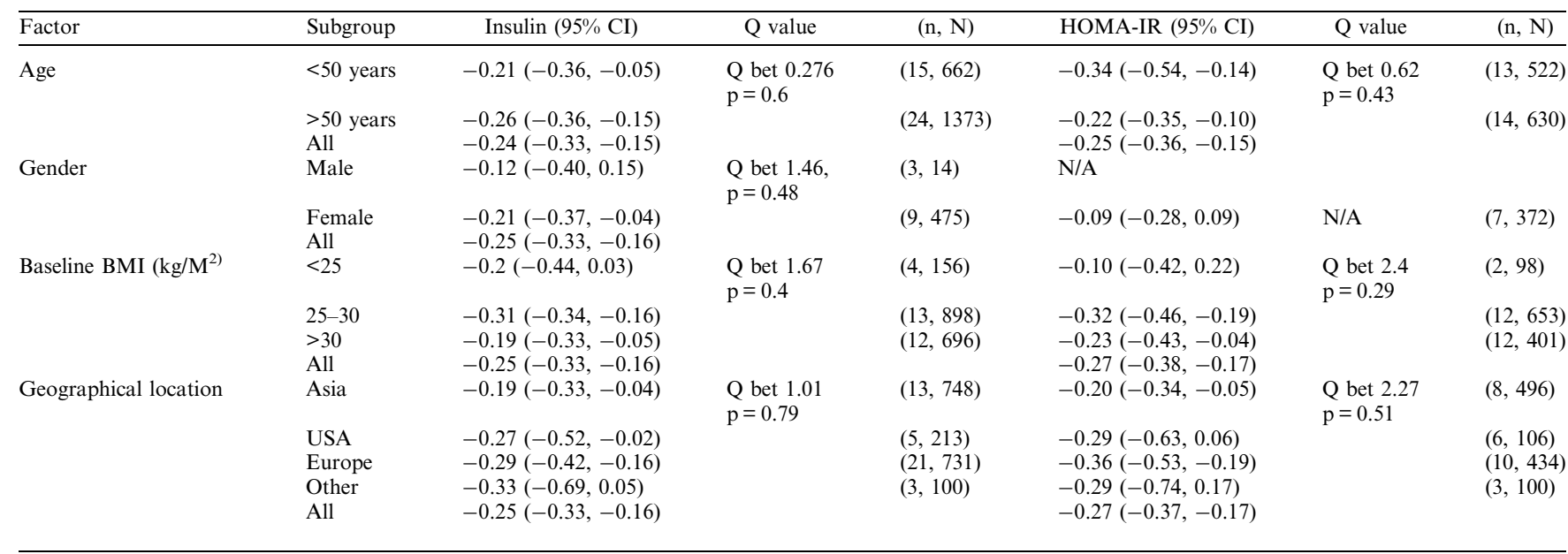

Values are showed in SMD $(95 \% \mathrm{CI}) . \mathrm{n}$, number of trials; N, number of participants.

Flavanols from tea, apple and cocoa were effective in modulating insulin and HOMA-IR. Inter-individual variability in the response was limited in contrast to previous studies ${ }^{(1,2)}$. This could be partly explained by the small number of trials reporting data for specific subgroups, and the broad range of doses and duration tested among the studies.

1. Pinto P \& Santos C. Eur J Nutr. 2017;56(4):1393-1408.

2. González-Sarrías A, Combet E, Pinto P et al. Nutrients. 2017;9(7):746. 